Research Article

\title{
Constrained Unscented Kalman Filter for Structural Identification of Bouc-Wen Hysteretic System
}

\author{
Sung-Yong Kim ${ }^{1}$ and Jaemin Kim $\mathbb{D}^{2}$ \\ ${ }^{1}$ School of Architecture, Changwon National University, Changwon, Republic of Korea \\ ${ }^{2}$ Department of Electronics Engineering, Myongji University, Yongin, Republic of Korea \\ Correspondence should be addressed to Jaemin Kim; jaemin@esl.mju.ac.kr
}

Received 8 May 2020; Revised 11 July 2020; Accepted 23 July 2020; Published 13 August 2020

Academic Editor: Daniele Baraldi

Copyright (C) 2020 Sung-Yong Kim and Jaemin Kim. This is an open access article distributed under the Creative Commons Attribution License, which permits unrestricted use, distribution, and reproduction in any medium, provided the original work is properly cited.

\begin{abstract}
The Bouc-Wen hysteresis model has been extensively used to identify earthquake damage on civil structures where hysteretic behavior is expected. One of the challenging problems in the identification of Bouc-Wen parameters is overfitting, which is caused by too many nonphysical model parameters. Limit on the search domain of parameters helps to solve the problem. The unscented Kalman filter (UKF) is widely developed to find Bouc-Wen parameters. In order to improve the accuracy and convergence speed of conventional UKF, this study developed a constrained UKF (CUKF) which can be used for the simultaneous identification of the Bouc-Wen hysteretic model. The proposed CUKF is very helpful when certain parameters can be constrained in a physical sense. We compare the proposed CUKF with conventional UKF to validate its robustness and efficacy in identifying Bouc-Wen parameters.
\end{abstract}

\section{Introduction}

Over the past few decades, several structural identification techniques have been successfully implemented for high-rise and long-span structures based on the development of computing and measurement technology. In the earthquake engineering field, structural identification techniques aim to quantify damage levels and guide potentially necessary immediate retrofitting efforts. Structural assessment in seismic engineering focuses on real-time hazard assessment using model updating techniques to find optimal parameters for structural models that can describe various complex nonlinear phenomena including yielding, postyielding, and strength and/or stiffness degradation. However, because it is difficult to describe complex inelastic behavior, monitoring of seismically affected structures is considered to be a challenging problem. Numerous models have been investigated for the description of nonlinear behaviors, and the Bouc-Wen model is one of the most popular models. It was originally introduced in [1] and extended in [2]. Owing to its versatility, the Bouc-Wen model has been successfully extended to create several variations, for assessing strength degradation, stiffness degradation [3], pinching effects [4-6], and asymmetric behavior [7-10]. It has also been widely adopted for the description of hysteretic structures [11-14].

The Kalman filter (KF) is widely used to estimate system states in practical applications, which consist of two consecutive processes called prediction and estimation. In the prediction process, the $\mathrm{KF}$ calculates predicted states and covariances, and in the following estimation process, the KF calculates Kalman gain based on the predicted covariances. The KF is widely used due to its ability in facilitating with only current measured values, previous states, and covariances for each iteration without requiring any additional previous information. The KF, however, cannot be used to analyze structures that are subjected to a severe earthquake because such structures exhibit nonlinear hysteretic behavior while the KF cannot be applied to a nonlinear system. To estimate the states of the nonlinear structures accurately, various numerical techniques have been investigated including the extended Kalman filter (EKF) [15-18], unscented Kalman filter (UKF) [19-26], and particle filter [27]. Among 
them, the UKF has an advantage in comparison to the EKF and particle filter: the UKF does not require Jacobians because they transform a set of points using known nonlinear equations and combine the results to estimate the mean and covariance of a state. Compared with particle filters that do not need Jacobians, the number of points used in a particle filter generally needs to be much greater than the number of points in a UKF.

However, as the demand for real-time applications has increased in many fields, the demand for technologies that provide accurate predictions in less computation has increased accordingly. Although the KF and its variations are powerful tools for state estimation on their own, they have the potential to facilitate faster and more accurate state estimation when using constraints on observed parameters identified by experts. Various studies have attempted to combine filtering techniques with nonlinear minimization algorithms [28-30] to facilitate the incorporation of prior knowledge regarding state parameters. In the health monitoring field, constrained dual EKFs are employed for the state estimation of linear structures. It has been proven numerically that constrained dual EKFs are efficient for state estimation. However, KFs with constraint functions have not yet been applied to nonlinear parameter estimation for the Bouc-Wen model.

This study implemented constrained minimization scheme in the use of UKF in order to improve the performance of the conventional UKF for real-time parameter identification. This paper first reviews the original Bouc-Wen model and several variations that have been proposed to simulate strength and strength degradation. Next, we propose a constrained UKF (CUKF) that combines a constrained minimization technique with a conventional UKF to determine the appropriate constraints for state and parameter identification. The proposed method is then compared with conventional UKF-based parameter identification techniques to validate its robustness and efficacy in identifying Bouc-Wen parameters.

\section{Parameter Identification via CUKF}

2.1. Problem Formulation. Let us consider a nonlinear dynamic system modeled as

$$
\begin{gathered}
x_{k+1}=F_{k}\left(x_{k}, \theta\right)+w_{k}, \\
y_{k}=H_{k}\left(x_{k}, \theta\right)+v_{k},
\end{gathered}
$$

where $x_{k} \in \mathbb{R}^{N}$ is the state variable, $y_{k} \in \mathbb{R}^{M}$ is the measured value, $\theta \in \mathbb{R}^{P}$ is the unknown parameter vector of interest, and $w_{k} \in \mathbb{R}^{N}$ and $v_{k} \in \mathbb{R}^{M}$ are additive process and sensor noises, respectively. We denote $F_{k}: \mathbb{R}^{N} \times \mathbb{R}^{P} \mapsto \mathbb{R}^{N}$ as the nonlinear vector-valued transition dynamics of the system and $H_{k}: \mathbb{R}^{N} \times \mathbb{R}^{P} \mapsto \mathbb{R}^{M}$ as a nonlinear vector-valued function, which transforms a state vector into the appropriate measurement vector. The subscript $k \in \mathbb{N}$ stands for the $k$-th discrete time step.

In order to estimate the unknown parameters, the unknown parameter vector $\theta$ is modeled as a discretized vector $\theta_{k}$, whose elements are the estimated parameters at the $k$-th step. We model the evolution of the estimated parameters as a Gaussian random walk process. An additional model for the parameter estimation can be considered as follows:

$$
\theta_{k+1}=\theta_{k}+\xi_{k}
$$

where $\xi_{k} \in \mathbb{R}^{P}$ is a zero-mean Gaussian white noise process. Here, equation (5) is reformulated by defining augmented vectors as $\tilde{x}_{k}=\left[x_{k} ; \theta_{k}\right]^{\mathrm{T}} \in \mathbb{R}^{N+P}, \widetilde{w}_{k}=\left[w_{k} ; \xi_{k}\right]^{\mathrm{T}} \in \mathbb{R}^{N+P}$, $\widetilde{F}_{k}: \mathbb{R}^{N+P} \mapsto \mathbb{R}^{N+P}$, and $\widetilde{H}_{k}: \mathbb{R}^{N+P} \mapsto \mathbb{R}^{M}$. An augmented system is formulated as follows:

$$
\begin{array}{r}
\tilde{x}_{k+1}=\widetilde{F}_{k}\left(\widetilde{x}_{k}\right)+\widetilde{w}_{k}, \\
y_{k}=\widetilde{H}_{k}\left(\tilde{x}_{k}\right)+v_{k} .
\end{array}
$$

We dispose the hat symbol for simplicity. We denote the total length of state vector as $N$ in this section.

2.2. Stage 1: Conventional Unscented Kalman Filter. UKF is a conventional method that attempts to estimate the state of the next step of the nonlinear system, whose posterior distribution is calculated by deterministic sampling approximation. We suppose that a state variable $x_{k} \in \mathbb{R}^{N}$ follows a normal distribution with its estimate $\widehat{x}_{k}$ and covariance $P_{k}$ denoted by $x_{k} \sim \mathcal{N}\left(\hat{x}_{k}, P_{k}\right)$. Under a conventional framework, a priori estimate and covariance for the next $k+1$ step are predicted by applying the following procedure.

2.2.1. Step 1: Initialization. At the $k$-th step, a set of sampling points is declared as

$$
\begin{aligned}
\chi_{1} & =\widehat{x}_{k}, \\
\chi_{i+1} & =\widehat{x}_{k}+\left(\sqrt{(N+\lambda) P_{k}}\right)_{i}, \quad i=1, \ldots, N, \\
\chi_{i+N+1} & =\widehat{x}_{k}-\left(\sqrt{(N+\lambda) P_{k}}\right)_{i}, \quad i=1, \ldots, N,
\end{aligned}
$$

where $\lambda=\sigma^{2}(N+\kappa)-N$ is a scaling parameter, $\sigma$ stands for spread of the sampling points around $\hat{x}_{k}, \kappa$ is a secondary scaling factor, and $(\sqrt{\cdot})_{i}$ denotes the $i$-th row of the matrix square root. The corresponding weight values $W_{i}$ for $i=$ $1, \ldots, N$ are declared as

$$
\begin{aligned}
W_{1} & =\frac{\lambda}{N+\lambda}, \\
W_{i+1} & =\frac{1}{2(N+\lambda)}, \quad i=1, \ldots, N, \\
W_{i+N+1} & =\frac{1}{2(N+\lambda)}, \quad i=1, \ldots, N .
\end{aligned}
$$

2.2.2. Step 2: Prediction. The projected mean and covariance are subsequently evaluated by using the projected sigma points as follows: 


$$
\begin{aligned}
& \hat{x}_{k+1}^{-}=\sum_{i=1}^{2 N+1} W_{i} F_{k}\left(\chi_{i}\right), \\
& P_{k+1}^{-}=\sum_{i=1}^{2 N+1} W_{i}\left\{F_{k}\left(\chi_{i}\right)-\widehat{x}_{k+1}^{-}\right\}\left\{F_{k}\left(\chi_{i}\right)-\widehat{x}_{k+1}^{-}\right\}^{\mathrm{T}}+Q_{k+1},
\end{aligned}
$$

where $Q_{k+1}$ is the process noise covariance at the $(k+1)$-th step. The projected sigma points are further substituted into the observation equation to generate the predicted system responses as follows:

$$
\begin{aligned}
& \widehat{y}_{k+1}^{-}=\sum_{i=1}^{2 N+1} W_{i} H_{k}\left(\chi_{i}\right) . \\
& P_{k+1}^{\mathrm{yy}}=\sum_{i=1}^{2 N+1} W_{i}\left\{H_{k}\left(\chi_{i}\right)-\widehat{y}_{k+1}^{-}\right\}\left\{H_{k}\left(\chi_{i}\right)-\widehat{y}_{k+1}^{-}\right\}^{\mathrm{T}}+R_{k+1},
\end{aligned}
$$

where $R_{k+1}$ is the output noise covariance at the $(k+1)$-th step.

2.2.3. Step 3: Correction. The Kalman gain $K_{k+1}$ at the $(k+1)$-th step is calculated as

$$
\begin{aligned}
P_{k+1}^{\mathrm{xy}} & =\sum_{i=1}^{2 N+1} W_{i}\left\{F_{k}\left(\chi_{i}\right)-\widehat{x}_{k+1}^{-}\right\}\left\{H_{k}\left(\chi_{i}\right)-\widehat{y}_{k+1}^{-}\right\}^{\mathrm{T}}, \\
K_{k+1} & =P_{k+1}^{\mathrm{xy}}\left(P_{k+1}^{\mathrm{yy}}\right)^{-1} .
\end{aligned}
$$

The estimated state $\hat{x}_{k+1}^{+}$and covariance $P_{k+1}^{+}$at the $(k+1)$-th step are finally calculated as

$$
\begin{aligned}
& \hat{x}_{k+1}^{+}=\hat{x}_{k+1}^{-}+K_{k+1}\left(y_{k+1}-\hat{y}_{k+1}^{-}\right), \\
& P_{k+1}^{+}=P_{k+1}^{-}-K_{k+1} P_{k+1}^{\mathrm{yy}} K_{k+1}^{\mathrm{T}} .
\end{aligned}
$$

A conventional UKF completes the estimation of the mean and covariance for the next step by calculating equations (11) and (12) as $\widehat{x}_{k+1}$ and $P_{k+1}$, respectively. The estimated parameters, however, may be not in a feasible region such as negative values' damping ratio or natural frequency. Such estimated parameters in the unacceptable range can result in lower accuracy in the estimation process or divergence problem. Hence, introducing an additional process that forces the corrected estimate $\hat{x}_{k+1}^{+}$into the feasible range can improve the estimation performance. The next stage adopts constrained minimization in order to project the corrected estimate to improve the estimation accuracy.

\subsection{Stage 2: Estimate Projection by Constrained Minimization.}

Having corrected an estimated state $\hat{x}_{k+1}^{+}$, a constrained estimate is obtained by projecting the unconstrained estimate onto a constraint surface. Interior-point methods are iterative schemes where the iterates approximate a local minimum from inside the feasible set. The constrained estimate can be obtained by solving the following minimization problem:

$$
\widehat{x}_{k+1}=\underset{x \in \Omega}{\operatorname{argmin}}\left(x-\widehat{x}_{k+1}^{+}\right)^{\mathrm{T}} S\left(x-\widehat{x}_{k+1}^{+}\right),
$$

where $\hat{x}_{k}$ and $\tilde{x}_{k}$ are the unconstrained estimate and constrained estimate of the state, respectively. We denote $S \in \mathbb{S}_{+}^{N}$ as a positive-definite weight matrix and $\Omega$ as the feasible region. A geometric illustration of estimate projection is presented in Figure 1, where the feasible set $\Omega$ is shaded and the contour lines of the convex quadratic objective function are depicted. It has been well established in previous studies [31] that setting $S$ to $I_{N \times N}$, where $I_{N \times N}$ is an identity matrix of size $N \times N$, results in the maximum probability estimate of the state. Setting $S$ to the covariance matrix $P_{k}$ calculated in equation (12) results in the least square estimate of the state. The estimate projection described in equation (13) is a constrained nonlinear function minimization that can typically be solved using well-established algorithms, including interiorpoint methods [32] and the active set method [33].

The advantage of introducing the constrained minimization technique in the parameter estimation problem is that the predicted parameters are bounded by the reasonable constraints. The constraints would make the UKF estimate more accurate compared with the conventional UKF estimate. Many practical problems require parameter estimation for physically meaningful constraints, which include positive natural frequencies, damping ratios, and energy dissipation. In the next section, the above method is applied to the state and parameter estimation of the Bouc-Wen model, which includes a severe nonlinearity, and the results thereof are discussed.

\section{Numerical Simulation}

3.1. Example 1: Original Bouc-Wen Hysteresis. A single-degree-of-freedom (SDOF) system with original Bouc-Wen hysteresis is considered in this example. It should be note that for the identification of earthquake damage, one specific element where a hysteretic behavior is expected, such as a column or a slender brace, is selected for observation to reduce the computational complexity: the overestimation problem may occur if measurement and identification are performed at many points including the point where elastic behavior is likely to occur. From the practical perspective, this study examined a SDOF system. The equation of motion for the example system when subjected to ground acceleration is written as

$$
\ddot{u}+2 \zeta_{n} \omega_{n} \dot{u}+\alpha \omega_{n}^{2} u+(1-\alpha) \omega_{n}^{2} z=-\ddot{u}_{g},
$$

where $u$ is the displacement, $\zeta_{n}$ is the damping ratio, $\omega_{n}$ is the natural frequency, $u_{g}$ is the ground displacement, $\alpha$ is the postyielding stiffness ratio, an overdot represents the time derivative, and $z$ is an auxiliary variable for describing the Bouc-Wen hysteresis governed by following differential equation:

$$
\dot{z}=\dot{u}\left[1-|z|^{n}\{\gamma+\beta \operatorname{sgn}(\dot{u} z)\}\right] .
$$

To perform parameter identification using the UKF, an augmented state vector is introduced as follows: 


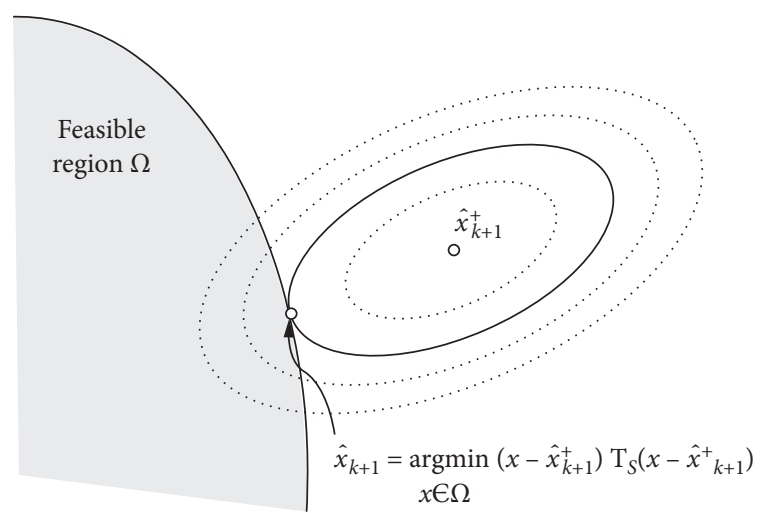

FIgURE 1: A geometric illustration of estimate projection diagram for constrained minimization of CUKF.

$$
x=[u, v, z, \alpha, \gamma, \beta, n]^{\mathrm{T}},
$$

where $v=\dot{u}$ is the velocity of the structure. Based on this augmented state vector, equations (14) and (15) can be reformulated into a state-space equation as follows:

$$
\dot{x}=f(x)=\left[\begin{array}{c}
v \\
-\ddot{u}_{g}-2 \zeta_{n} \omega_{n} v-\alpha \omega_{n}^{2} u+(1-\alpha) \omega_{n}^{2} z \\
v\left[1-|z|^{n}\{\gamma+\beta \operatorname{sgn}(v z)\}\right] \\
O_{4 \times 1}
\end{array}\right],
$$

where $O_{N \times M} \in \mathbb{R}^{N \times M}$ is a zero matrix. In this example, the noise term for the state variable in equation (2) is neglected. Additionally, the damping ratio and natural frequency are discarded from the augmented vector because it is assumed that they were previously identified under ambient conditions. This point will be discussed in detail in the next example. We choose absolute acceleration as the measurement variable, which is common in real-world application. The output equation for the measurement can be written as follows:

$$
y=\ddot{u}+\ddot{u}_{g}+w=-2 \zeta_{n} \omega_{n} v-\alpha \omega_{n}^{2} u-(1-\alpha) \omega_{n}^{2} z+\ddot{u}_{g}+w,
$$

where $w$ is the measurement noise. A Gaussian white noise process with root mean square (RMS) value of $1 \mathrm{~m} / \mathrm{s}^{2}$ is considered based on its relatively large value in the context of the general measurement procedure. We choose the ground motion of the $1940 \mathrm{El}$ Centro earthquake as the input excitation. It has a $50 \mathrm{~Hz}$ sampling rate and a 30 -second duration. To perform system identification using the Kalman filter methods, we reformulate equations (17) and (18) into a discretized form of equation (2) using the fourth-order Runge-Kutta integration method. The initial values of the parameters $u$ and $v$ in the space vector $x$ are set to zero. Table 1 lists the true model parameters and initial guessed model parameters for this example. The postyielding parameter $\alpha$ is set to 0.09 , which is a practical value for steel. The shape parameters $n, \beta$, and $\gamma$ are set to $1.2,20$, and 40 , respectively. The initial guesses are determined to have the same or a similar order of magnitude compared to the corresponding true values. We configure the initial covariance matrix $P$ in diagonal matrix form to ensure that the designed filter could be applied to a roughly estimated condition as follows:

$$
P=\operatorname{diag}\left(10^{-3}, 10^{-3}, 10^{-3}, 10^{-1}, 10^{3}, 10^{3}, 10^{-1}\right),
$$

where $\operatorname{diag}(\cdot)$ is a diagonal matrix with arguments along the main diagonal. To utilize the CUKF, the following constraint conditions are determined based on physical intuition. First, the parameters $\alpha, \gamma, \beta$, and $n$ are constrained to be positive. Second, the upper bound of the stiffness ratio $\alpha$ is set to 0.2 . Finally, $\beta+\gamma$ is bounded in the range of 35-70, meaning the limit value $z_{m}$ should be bounded in a range of $0.01-0.03$ if the parameter $n$ is near unity. The constraint conditions onto which the estimated state will be projected constitute the following feasible region:

$$
\Omega=\left\{x \in \mathbb{R}^{7}: A x<b\right\},
$$

where

$$
\begin{aligned}
A & =\left[\begin{array}{ccccccc}
0 & 0 & 0 & -1 & 0 & 0 & 0 \\
0 & 0 & 0 & 0 & -1 & 0 & 0 \\
0 & 0 & 0 & 0 & 0 & -1 & 0 \\
0 & 0 & 0 & 0 & 0 & 0 & -1 \\
0 & 0 & 0 & 0 & -1 & -1 & 0 \\
0 & 0 & 0 & 0 & 1 & 1 & 0
\end{array}\right], \\
b & =\left[\begin{array}{c}
0 \\
0 \\
0 \\
0 \\
-35 \\
70
\end{array}\right] .
\end{aligned}
$$

Therefore, the procedure for finding the constrained estimate $\hat{x}_{k}$ defined in equation (13) is a linearly constrained quadratic optimization problem. A variety of numerical tools can solve such problems. A MATLAB function called quadprog with an interior-point-convex algorithm is employed to solve the constrained quadratic problem in this example.

Figure 2 presents a comparison of the accelerations estimated by the UKF and CUKF. It is evident that the acceleration histories estimated by the UKF and CUKF correlate with the true simulated responses such that they cannot be distinguished from the true responses. Their absolute errors reach a maximum of $0.5\left(\mathrm{~m} / \mathrm{s}^{2}\right)$.

Figure 3 presents the time histories of the parameters estimated by the UKF and CUKF. Table 1 summarizes the final estimated values for the parameters. By carefully inspecting the time histories for identifying parameter $\alpha$, one can see that the UKF attempts to seek a value of $\alpha$ larger than 0.3 at approximately $1 \mathrm{~s}$ and $4 \mathrm{~s}$, which is an infeasible region from a physical perspective. In contrast, the CUKF estimates parameters closer to the true values compared with the UKF by searching for the parameters within admissible physical constraints. The inaccuracy of parameters combined with good agreement with predicted measurements indicates overfitting for the UKF. The parameters of the Bouc-Wen 
TABle 1: Model parameters used in Example 1.

\begin{tabular}{lcccrc}
\hline Parameter & True & Initial guess & UKF & Estimated & \multicolumn{2}{c}{ Absolute error } \\
CUKF
\end{tabular}

${ }^{\dagger}$ These parameters are presumed to be determined.

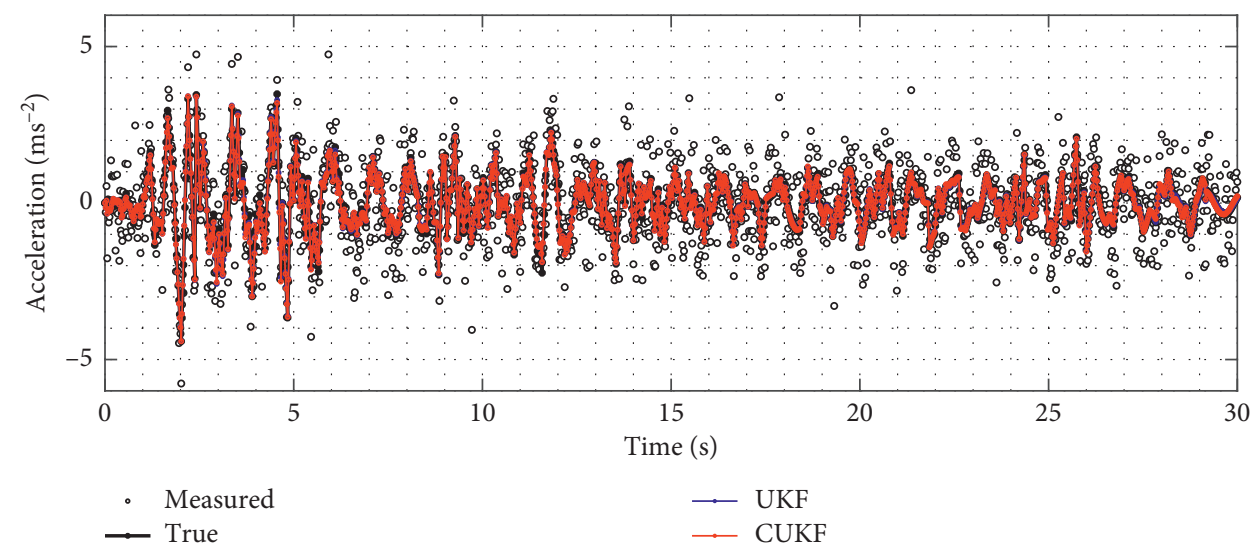

(a)

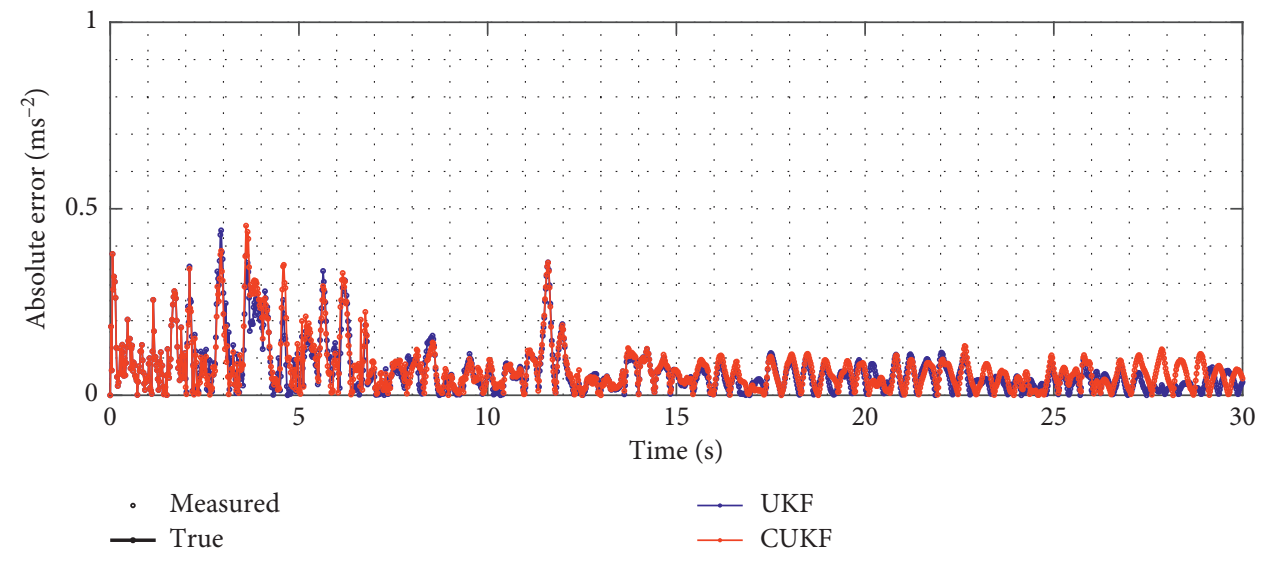

(b)

FIgURE 2: Comparison of acceleration time histories identified by UKF and CUKF (a) and their absolute errors (b) for Example 1.

model are empirical, meaning they are not derived from fundamental mechanics, and a given response may not determine parameters unambiguously [34]. Therefore, it is helpful for applying the CUKF to system parameter identification if certain parameters can be constrained in a physical sense. It can be seen that the proposed CUKF can provide accurate results for estimation of all parameters except for the parameter $n$, which shows a large error of about $4 \%$. However, when we recall from the previous studies on the parameters of the Bouc model that the variable $n$ only describes the sharpness of hysteretic behavior (for example, refer to [34]), it can be seen that the estimation results accurately predict the stiffness and strength of the hysteretic behavior.
3.2. Example 2: Overestimation Problem. To test the overestimation problem, an additional simulation is conducted in which the structure examined in the previous example is subjected to ambient ground motion. In the example, the governing equation is identical to that for Example 1, but the structure is assumed to be subjected to the ground motion of the $1940 \mathrm{El}$ Centro earthquake at a scale of $1 / 10$. We assume that the structure is elastic and the Bouc-Wen parameters should have no influence on structural behavior. The augmented vector includes the damping ratio and natural frequency. We define the augmented state vector $x$ as follows:

$$
x=\left[u, v, z, \alpha, \gamma, \beta, n, \omega_{n}, \zeta_{n}\right]^{\mathrm{T}} .
$$



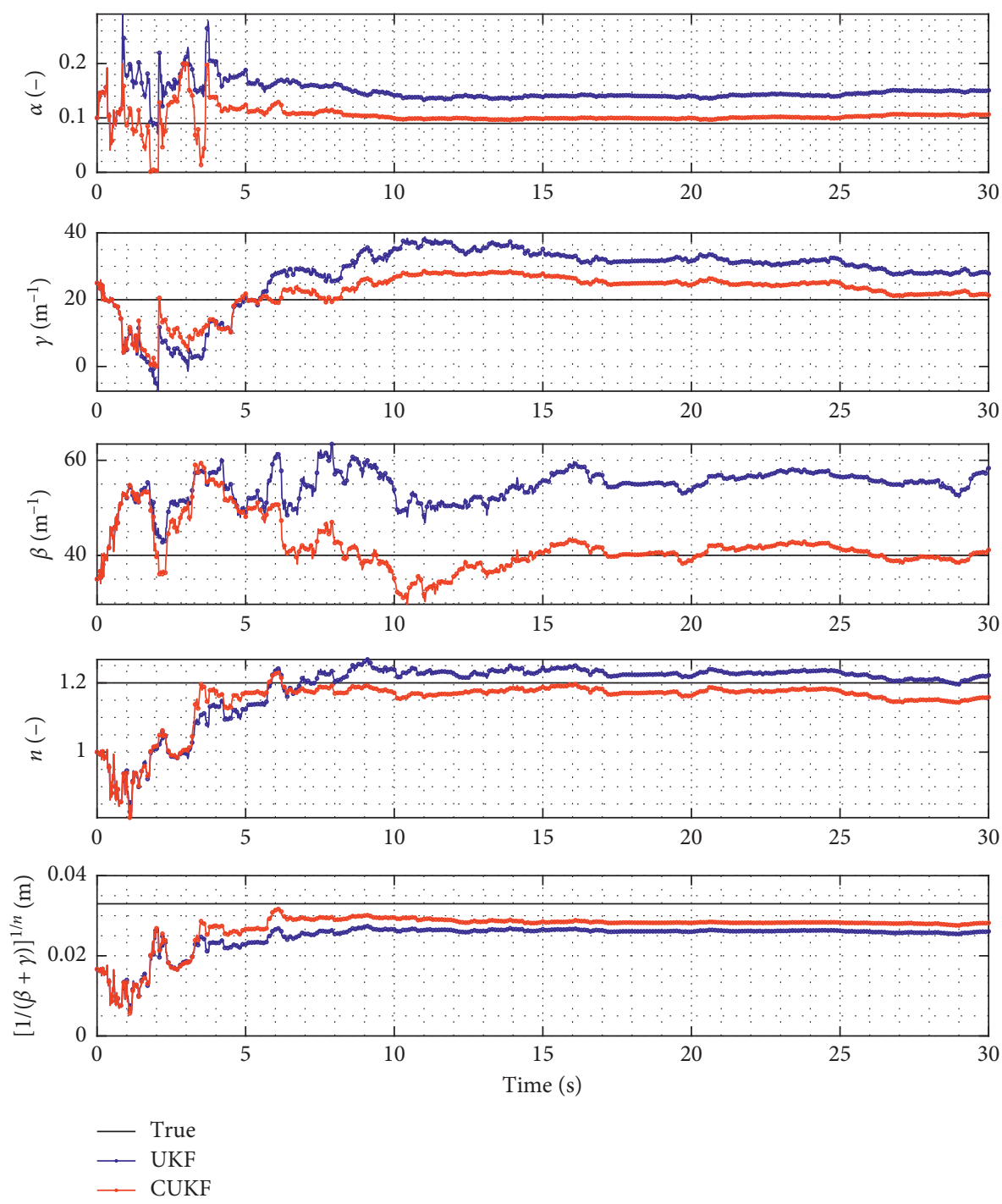

FIgURE 3: Results of parameter identification in Example 1.

TABle 2: Model parameters used in Example 2.

\begin{tabular}{|c|c|c|c|c|c|c|c|c|}
\hline \multirow{2}{*}{ Parameter } & \multirow{2}{*}{ True } & \multirow{2}{*}{ Initial guess } & \multicolumn{3}{|c|}{ Estimated } & \multicolumn{3}{|c|}{ Absolute error } \\
\hline & & & UKF & CUKF1 & CUKF2 & UKF & CUKF1 & CUKF2 \\
\hline$\zeta_{n}(\%)$ & 2 & 3 & -3.48 & 3.68 & 2.78 & 5.48 & 1.68 & 0.78 \\
\hline$\omega_{n}(\mathrm{rad} / \mathrm{s})$ & $2 \pi$ & $2.4 \pi$ & -4.20 & 6.28 & 6.33 & 10.48 & 0.00 & 0.05 \\
\hline$\alpha\left(\times 10^{-2}\right)$ & 9 & 10 & 33.94 & 5.25 & $--^{\dagger}$ & 24.94 & 3.75 & $--^{\dagger}$ \\
\hline$\gamma\left(\times 10 \mathrm{~m}^{-1}\right)$ & 2 & 2.5 & -2.52 & 4.64 & $-^{\dagger}$ & 4.52 & 2.64 & $-^{\dagger}$ \\
\hline$\beta\left(\times 10 \mathrm{~m}^{-1}\right)$ & 4 & 3.5 & 0.13 & 2.19 & $-^{\dagger}$ & 3.87 & 1.81 & $-^{\dagger}$ \\
\hline$n(-)$ & 1.2 & 1 & 0.96 & 1.50 & $--^{\dagger}$ & 0.30 & 0.24 & $-^{\dagger}$ \\
\hline
\end{tabular}

${ }^{\dagger}$ These values are undefined because they are constrained by equality constraints.

A Gaussian white noise process with an RMS value of $0.1 \mathrm{~m} / \mathrm{s}^{2}$ is applied to the measurements. Just as in the previous example, the initial values for the original state vector $x$ are set to zero. Table 2 lists the true and initially guessed model parameters for this example. The true natural frequency and damping ratio are $2 \pi$ and 0.02 , respectively, which are same as the corresponding values in Example 1. The postyielding parameter $\alpha$ and shape parameters $n, \beta$, and $\gamma$ are set to $0.09,1.2,20$, and 40 , respectively.
Two CUKFs are utilized in the example. CUKF1 is identical to the CUKF used in Example 1, except that the natural frequency and damping ratio are included in the state vector and constrained to be positive. CUKF2 is a constrained UKF that includes several equality constraints based on physical intuition. In CUKF2, to suppress the Bouc-Wen parameters during the identification process, the parameters $\alpha, \gamma, \beta$, and $n$ are set to be constrained to $0.1,1$, 25 , and 35 , respectively. The constraint conditions onto 


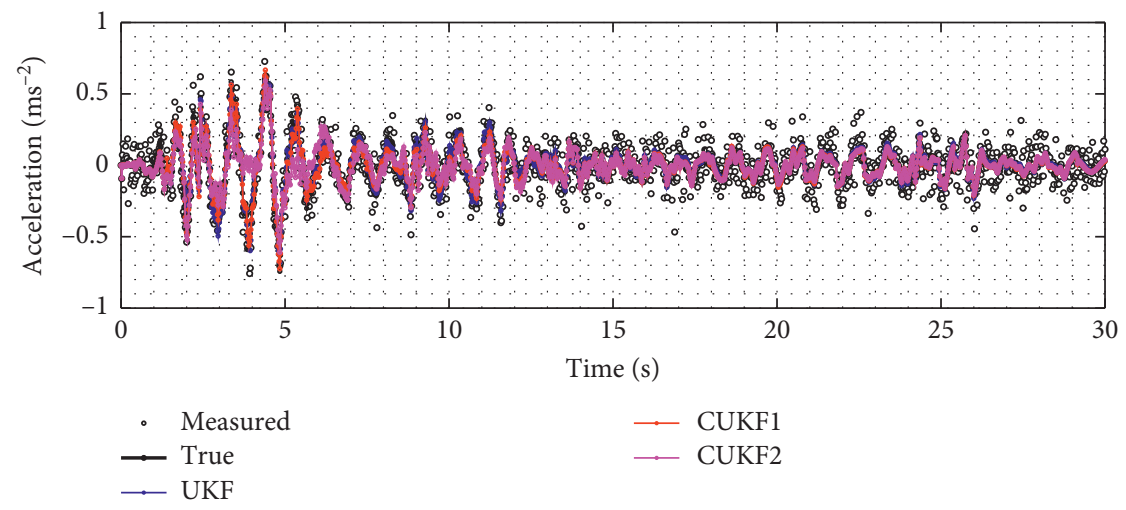

FIGURE 4: Comparison of acceleration time history results for Example 2 identified by the UKF, CUKF1, and CUKF2.

which the estimated state is projected constitute the following feasible region:

$$
\Omega=\left\{x \in \mathbb{R}^{9}: A x<b \text { and } A_{\mathrm{eq}} x=b_{\mathrm{eq}}\right\},
$$

where

$$
\begin{aligned}
& A=\left[\begin{array}{ccccccccc}
0 & 0 & 0 & -1 & 0 & 0 & 0 & 0 & 0 \\
0 & 0 & 0 & 0 & -1 & 0 & 0 & 0 & 0 \\
0 & 0 & 0 & 0 & 0 & -1 & 0 & 0 & 0 \\
0 & 0 & 0 & 0 & 0 & 0 & -1 & 0 & 0 \\
0 & 0 & 0 & 0 & 0 & 0 & 0 & -1 & 0 \\
0 & 0 & 0 & 0 & 0 & 0 & 0 & 0 & -1 \\
0 & 0 & 0 & 0 & -1 & -1 & 0 & 0 & 0 \\
0 & 0 & 0 & 0 & 1 & 1 & 0 & 0 & 0
\end{array}\right], \\
& b=\left[\begin{array}{c}
0 \\
0 \\
0 \\
0 \\
0 \\
0 \\
-35 \\
70
\end{array}\right] . \\
& A_{\text {eq }}=\left[\begin{array}{lllllllll}
0 & 0 & 0 & 1 & 0 & 0 & 0 & 0 & 0 \\
0 & 0 & 0 & 0 & 1 & 0 & 0 & 0 & 0 \\
0 & 0 & 0 & 0 & 0 & 1 & 0 & 0 & 0 \\
0 & 0 & 0 & 0 & 0 & 0 & 1 & 0 & 0
\end{array}\right], \\
& b_{\text {eq }}=\left[\begin{array}{c}
10 \\
25 \\
35 \\
1
\end{array}\right] \text {. }
\end{aligned}
$$

Figure 4 presents a comparison of the accelerations estimated by the UKF, CUKF1, and CUKF2. The acceleration histories estimated by the UKF and CUKFs correlate well with the true simulated responses. Figure 5 shows the time histories of the parameters estimated by the UKF and CUKFs. The UKF attempts to seek the values of $\omega_{n}$ and $\zeta_{n}$ smaller than zero, which is infeasible from a physical perspective, resulting in overestimation. In contrast, both CUKFs estimate these parameters accurately by searching within a positive space. CUKF1 forcibly predicts the Bouc-Wen parameters, which are unnecessary for describing the elastic behavior of the examined structure. Therefore, as noted in the previous example, it is very helpful for applying the CUKF to system parameter identification if some parameters can be constrained in a physical sense. We summarize the final values estimated by the UKF and CUKFs in Table 2, which indicates that the CUKFs are superior for estimating accurate parameters.

3.3. Example 3: Structure with Degrading Hysteresis. The final example uses the SDOF structure from Example 1, but degrading effects are included. In the model proposed by [4], the auxiliary variable $z$ in equation (15) is modified in order to employ strength and stiffness degradation as follows:

$$
\dot{z}=\frac{\dot{u}\left[1-\nu|z|^{n}\{\gamma+\beta \operatorname{sgn}(\dot{u} z)\}\right]}{\eta},
$$

where the parameters $v$ and $\eta$ are expressed as linear functions of the dissipated energy and their slopes $\delta_{\nu}$ and $\delta_{\eta}$ such as

$$
\begin{aligned}
& \nu=1+\delta_{\nu} \varepsilon, \\
& \eta=1+\delta_{\eta} \varepsilon,
\end{aligned}
$$

with $\varepsilon$ being the dissipated hysteretic energy as

$$
\varepsilon=\varepsilon(t)=(1-\alpha) \omega_{n}^{2} \int_{0}^{t} z \dot{u} \mathrm{~d} t .
$$

An augmented state vector is introduced to formulate the state-space equation as follows:

$$
x=\left[u, v, z, \varepsilon, \alpha, \gamma, \beta, n, \delta_{v}, \delta_{\eta}\right]^{\mathrm{T}} .
$$

Using this augmented state vector, equations (25), and (26) are reformulated as the following nonlinear state-space equation: 

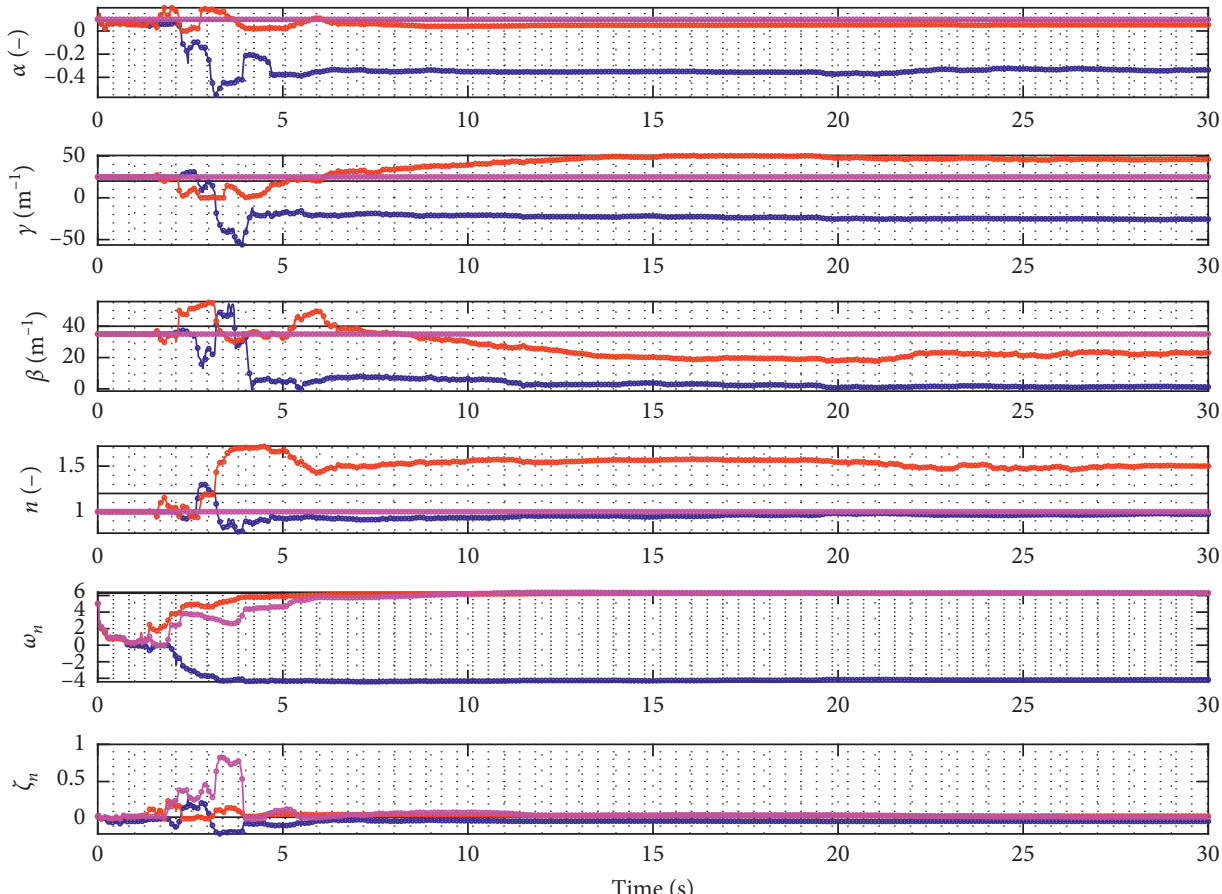

— True

$\longrightarrow$ CUKF1

— UKF

$\because$ CUKF2

Figure 5: Results of parameter identification for Example 2.

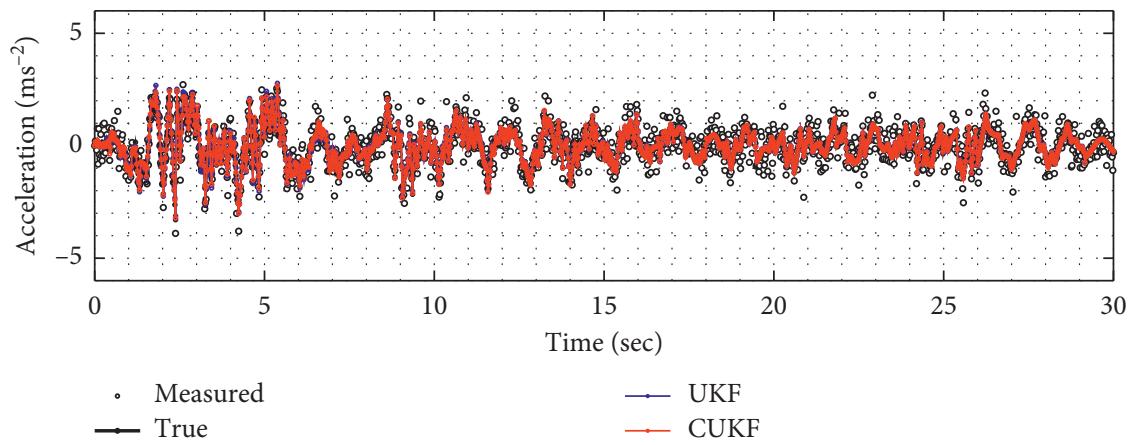

(a)

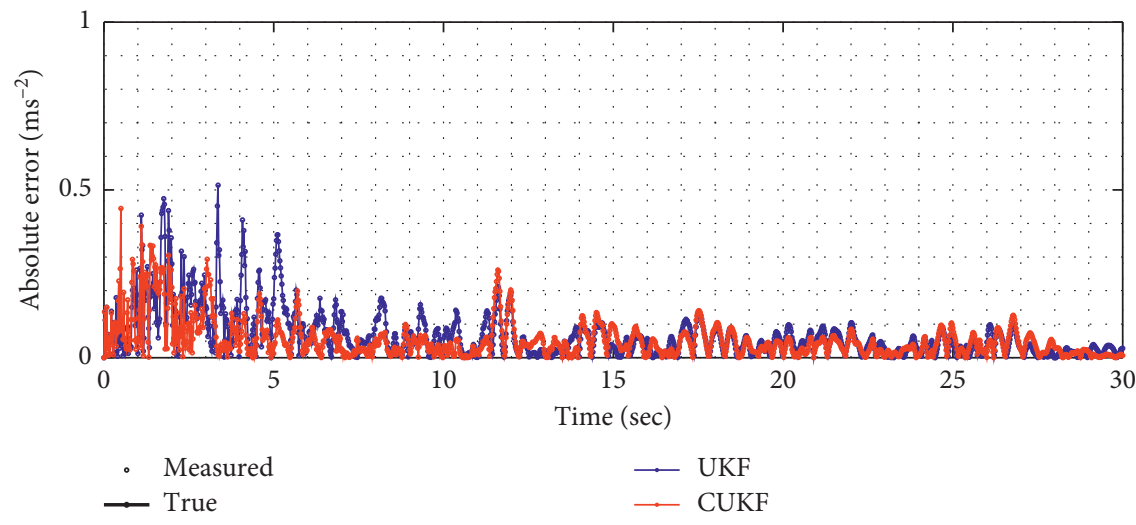

(b)

FIGURE 6: Comparison of acceleration time history results for Example 3 identified by the UKF and CUKF (a) and the corresponding absolute errors (b). 

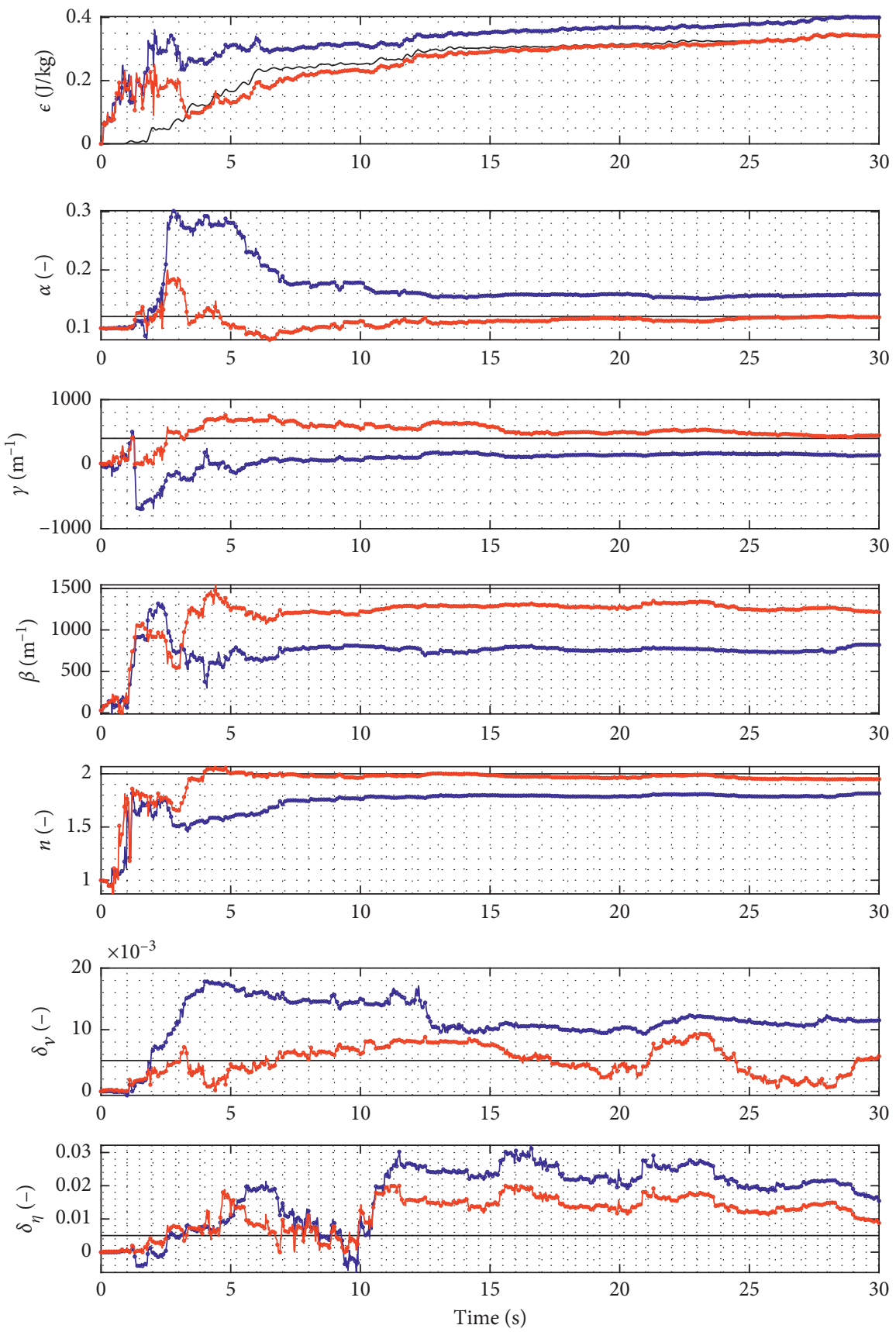

$\longrightarrow$ True

$\rightarrow$ CUKF

Figure 7: Results of parameter identification for Example 3.

TABle 3: Model parameters used in Example 3.

\begin{tabular}{|c|c|c|c|c|c|c|}
\hline \multirow{2}{*}{ Parameter } & \multirow{2}{*}{ True } & \multirow{2}{*}{ Initial guess } & \multicolumn{2}{|c|}{ Estimated } & \multicolumn{2}{|c|}{ Absolute error } \\
\hline & & & UKF & CUKF & UKF & CUKF \\
\hline$\zeta_{n}^{\dagger}(\%)$ & 2 & - & - & - & - & - \\
\hline$\omega_{n}^{\dagger}(\mathrm{rad} / \mathrm{s})$ & $2 \pi$ & - & - & - & - & - \\
\hline$\alpha^{n}\left(\times 10^{-2}\right)$ & 12 & 10 & 16.96 & 11.33 & 4.96 & 1.33 \\
\hline$\gamma\left(\times 10^{2} \mathrm{~m}^{-1}\right)$ & 4 & 0.1 & 0.79 & 5.03 & 0.77 & 0.24 \\
\hline$\beta\left(\times 10^{2} \mathrm{~m}^{-1}\right)$ & 15 & 0.3 & 7.44 & 11.94 & 7.56 & 3.06 \\
\hline$n(-)$ & 2 & 1 & 1.73 & 1.93 & 0.27 & 0.07 \\
\hline$\delta_{\nu}\left(\times 10^{-2}\right)$ & 0.5 & 0 & 1.2 & 0.5 & 0.7 & 0 \\
\hline$\delta_{\eta}\left(\times 10^{-2}\right)$ & 0.5 & 0 & 1.7 & 1.2 & 1.2 & 0.7 \\
\hline
\end{tabular}

\footnotetext{
${ }^{\dagger}$ These parameters are presumed to be determined.
} 


$$
\dot{x}=f(x)=\left[\begin{array}{c}
v \\
-\ddot{u}_{g}-2 \zeta_{n} \omega_{n} v-\alpha \omega_{n}^{2} u-(1-\alpha) \omega_{n}^{2} z \\
\frac{v}{\eta}\left[1-v|z|^{n}\{\gamma+\beta \operatorname{sgn}(v z)\}\right] \\
z v \\
O_{6 \times 1}
\end{array}\right] .
$$

Figure 6 presents a comparison of the accelerations estimated by the UKF and CUKF. The acceleration histories estimated by the UKF and CUKF correlate well with the true simulated responses, and they cannot be distinguished from the true responses. Their absolute errors reach a maximum of only $0.5\left(\mathrm{~m} / \mathrm{s}^{2}\right)$. Figure 7 shows the time histories of the parameters estimated by the UKF and CUKF. The final estimated values for the parameters are summarized in Table 3. By carefully inspecting the time histories for identifying parameter $\alpha$, the UKF attempts to seek a value greater than 0.3 at approximately $1 \mathrm{~s}$ and $4 \mathrm{~s}$, which is unacceptable. In contrast, the CUKF estimates parameters closer to the true values compared to the UKF by searching for parameters within constraints. The estimated values in Table 3 are maximum likelihood estimates for the parameters of a normal distribution based on the estimated sample sequences from the simulations.

\section{Summary and Conclusions}

Parameter identification of the structure is challenging in the seismic engineering field due to its complex nonlinear behavior. The Bouc-Wen hysteresis model is the most widely used model to identify the parameter. However, it can suffer from overfitting problems when measurements are limited in practice. We adopt the constrained unscented Kalman filter (CUKF) for the state and parameter estimation problem of the Bouc-Wen model to overcome the overfitting problems. The applied CUKF combines a constrained minimization technique with a conventional UKF. The predicted parameters are bounded for the reasonable constraints, which make the UKF estimate more accurate compared with the conventional UKF. We validate the CUKF by exhibiting several numerical examples. We finally show that the proposed CUKF is very helpful, robust, and efficient for system parameter identification by the comparative result when certain parameters are constrained in a physical sense.

\section{Data Availability}

The data used to support the findings of the study are available from the corresponding author upon request.

\section{Conflicts of Interest}

The authors declare that they have no conflicts of interest.

\section{Acknowledgments}

This work was supported by the National Research Foundation of Korea (NRF) grant funded by the Korea government (MSIT) (No. 2018R1C1B6009196) and the 2019 Research Fund of Myongji University.

\section{References}

[1] R. Bouc, "Forced vibrations of mechanical systems with hysteresis," in Proceedings of the Fourth Conference on Nonlinear Oscillations, Prague, September 1967.

[2] Y.-K. Wen, "Method for random vibration of hysteretic systems," Journal of the Engineering Mechanics Division, vol. 102, no. 2, pp. 249-263, 1976.

[3] A. K. Kottari, A. E. Charalampakis, and V. K. Koumousis, "A consistent degrading Bouc-Wen model," Engineering Structures, vol. 60, pp. 235-240, 2014.

[4] T. T. Baber and Y.-K. Wen, "Random vibration of hysteretic degrading systems," Journal of the Engineering Mechanics Division, vol. 107, no. 6, pp. 1069-1087, 1981.

[5] T. T. Baber and M. N. Noori, "Random vibration of degrading, pinching systems," Journal of Engineering Mechanics, vol. 111, no. 8, pp. 1010-1026, 1985.

[6] G. C. Foliente, "Hysteresis modeling of wood joints and structural systems," Journal of Structural Engineering, vol. 121, no. 6, pp. 1013-1022, 1995.

[7] W. Zhu and D.-H. Wang, "Non-symmetrical bouc-wen model for piezoelectric ceramic actuators," Sensors and Actuators A: Physical, vol. 181, pp. 51-60, 2012.

[8] J. Gan and X. Zhang, "An enhanced bouc-wen model for characterizing rate-dependent hysteresis of piezoelectric actuators," Review of Scientific Instruments, vol. 89, no. 11, p. 115002, 2018.

[9] G. Wang, G. Chen, and F. Bai, "Modeling and identification of asymmetric bouc-wen hysteresis for piezoelectric actuator via a novel differential evolution algorithm," Sensors and Actuators A: Physical, vol. 235, pp. 105-118, 2015.

[10] S.-Y. Kim and C.-h. Lee, "Description of asymmetric hysteretic behavior based on the bouc-wen model and piecewise linear strength-degradation functions," Engineering Structures, vol. 181, pp. 181-191, 2019.

[11] N. Kwok, Q. Ha, T. Nguyen, J. Li, and B. Samali, "Bouc-Wen model parameter identification for a MR fluid damper using computationally efficient GA," ISA Transactions, vol. 46, no. 2, pp. 167-179, 2007.

[12] X. Zhu and X. Lu, "Parametric identification of bouc-wen model and its application in mild steel damper modeling," Procedia Engineering, vol. 14, pp. 318-324, 2011.

[13] T. Sireteanu, M. Giuclea, A.-M. Mitu, and G. Ghita, "A genetic algorithms method for fitting the generalized Bouc-Wen model to experimental asymmetric hysteretic loops," Journal of Vibration and Acoustics, vol. 4, Article ID 041007, 2012.

[14] M. Y. Ye, H. Jiang, Y. S. Xu, and X. D. Wang, "Bouc-wen hysteresis model parameter identification by means of hybrid intelligent technique," Advanced Materials Research, vol. 108, pp. 1397-1402, 2010.

[15] H. Ebrahimian, R. Astroza, and J. P. Conte, "Extended Kalman filter for material parameter estimation in nonlinear structural finite element models using direct differentiation method," Earthquake Engineering \& Structural Dynamics, vol. 44, pp. 1495-1522, 2015, http://arxiv.org/abs/1403.5481.

[16] Z. Zhou, L. Zhao, L. Xie, S. Xue, H. Tang, and C. Wan, "Parameter identification for structural health monitoring 
with extended kalman filter considering integration and noise effect," Applied Sciences, vol. 8, no. 12, p. 2480, 2018.

[17] S. Sen and B. Bhattacharya, "Online structural damage identification technique using constrained dual extended Kalman filter," Structural Control and Health Monitoring, vol. 24, pp. 1-12, 2016.

[18] M. N. Chatzis, E. N. Chatzi, and S. P. Triantafyllou, "A discontinuous extended kalman filter for non-smooth dynamic problems," Mechanical Systems and Signal Processing, vol. 92, pp. 13-29, 2017.

[19] J. H. Gove and D. Y. Hollinger, "Application of a dual unscented Kalman filter for simultaneous state and parameter estimation in problems of surface-atmosphere exchange," Journal of Geophysical Research, vol. 111, pp. 1-21, 2006.

[20] M. Wu and A. W. Smyth, "Real-time parameter estimation for degrading and pinching hysteretic models," International Journal of Non-linear Mechanics, vol. 43, no. 9, pp. 822-833, 2008.

[21] E. N. Chatzi and A. W. Smyth, "The unscented kalman filter and particle filter methods for nonlinear structural system identification with non-collocated heterogeneous sensing," Structural Control and Health Monitoring: The Official Journal of the International Association for Structural Control and Monitoring and of the European Association for the Control of Structures, vol. 16, no. 1, pp. 99-123, 2009.

[22] R. Omrani, R. Hudson, and E. Taciroglu, "Parametric identification of nondegrading hysteresis in a laterally and torsionally coupled building using an unscented kalman filter," Journal of Engineering Mechanics, vol. 139, no. 4, pp. 452-468, 2012.

[23] M. Wu and A. W. Smyth, "Application of the unscented Kalman filter for real-time nonlinear structural identification," Structural Control and Health Monitoring, vol. 14, pp. 971-990, 2007.

[24] E. N. Chatzi, A. W. Smyth, and S. F. Masri, "Experimental application of on-line parametric identification for nonlinear hysteretic systems with model uncertainty," Structural Safety, vol. 32, no. 5, pp. 326-337, 2010.

[25] Z. Xie and J. Feng, "Real-time nonlinear structural system identification via iterated unscented Kalman filter," $\mathrm{Me}$ chanical Systems and Signal Processing, vol. 28, no. 20100480049, pp. 309-322, 2012.

[26] W. Song and S. Dyke, "Real-time dynamic model updating of a hysteretic structural system," Journal of Structural Engineering, vol. 140, no. 3, pp. 1-14, 2013.

[27] N. Kwok, Q. Ha, T. Nguyen, J. Li, and B. Samali, “A novel hysteretic model for magnetorheological fluid dampers and parameter identification using particle swarm optimization," Sensors and Actuators A: Physical, vol. 132, no. 2, pp. 441-451, 2006.

[28] N. Gupta and R. Hauser, "Kalman filtering with equality and inequality state constraints," http://arxiv.org/abs/0709.2791. Tech. Rep. 07/18, Oxford University, Oxford, UK, 2007, http://arxiv.org/abs/0709.2791. Tech. Rep. 07/18.

[29] R. Kandepu, B. Foss, and L. Imsland, "Applying the unscented Kalman filter for nonlinear state estimation," Journal of Process Control, vol. 18, no. 7-8, pp. 753-768, 2008.

[30] D. J. Simon, "Kalman filtering with state constraints: a survey of linear and nonlinear algorithms," IET Control Theory and Applications, vol. 4, 2010.

[31] D. Simon and T. L. Chia, "Kalman filtering with state equality constraints," IEEE Transactions on Aerospace and Electronic Systems, vol. 38, no. 1, pp. 128-136, 2002.
[32] S. Boyd and L. Vandenberghe, Convex optimization, Cambridge University Press, Cambridge, UK, 2004.

[33] J. Nocedal and S. Wright, Numerical optimization, Springer Science \& Business Media, Berlin, Germany, 2006.

[34] F. Ma, H. Zhang, A. Bockstedte, G. C. Foliente, and P. Paevere, "Parameter analysis of the differential model of hysteresis," Journal of Applied Mechanics, vol. 71, no. 3, p. 342, 2004. 